\title{
Estimating Transonic Drag
}

\author{
$\begin{array}{lll}\text { B. J. Florio } & \text { R. McKibbin } & \\ & \text { C. Braham } & \end{array}$ \\ J. R. Ockendon ${ }^{4} \quad$ C. P. Please ${ }^{5}$
}

(Received 17 July 2018; revised 19 May 2019)

\begin{abstract}
To predict the trajectory of projectiles such as bullets and mortar shells, we require knowledge of the acting forces, including drag. For the most part, the drag coefficient (which is dependent on the local Mach number) is well-understood for subsonic and supersonic velocities. However, there is often a rapid and unintuitive change in the drag behaviour near the speed of sound. The transonic behaviour of the projectiles is addressed in two major ways. First we explain the underlying physics of drag in the three major regimes. The appearance of shock waves alters the drag forces dramatically. In some situations, the physical models are simplified to directly obtain the drag coefficient profile. Then we tackle an inverse problem, where firing table data gives the drag coefficient profile. The drag profile obtained by both point-by-point optimisation and by parametrising a suitable family of functions. Finally, transonic data is difficult to obtain in wind-tunnel
\end{abstract}

DOI:10.21914/anziamj.v59i0.13408, C) Austral. Mathematical Soc. 2019. Published 2019-06-21, as part of the Proceedings of the 2017 Mathematics and Statistics in Industry Study Group. ISSN 1445-8810. (Print two pages per sheet of paper.) Copies of this article must not be made otherwise available on the internet; instead link directly to the DOI for this article. 
experiments, so based on our understanding of the physics, alternative experiments are suggested. This research was undertaken as part of the 2017 Mathematics in Industry Study Group (Adelaide) with industry partner, the Defence Science and Technology group.

\section{Contents}

1 Introduction

2 The physics of steady aerodynamics M5

2.1 Subsonic flow $(\mathrm{M}<1) \ldots \ldots \ldots \ldots$ M6 . . . . . . . . .

2.2 Supersonic flow $(M>1) \ldots \ldots \ldots$ M8

2.3 Transonic flow $(\mathrm{M} \approx 1) \ldots \ldots \ldots$ M9

3 Low angle approximations to the projectile path $\quad$ M11

4 Attraction to Mach $1 \quad$ M14

5 Possible approximations to the drag coefficient $\quad$ M19

5.1 Functional forms for $\mathrm{C}_{\mathrm{d}} \ldots \ldots \ldots \ldots$ M20

5.2 Optimising with the three-parameter hyperbolic tangent . M22

5.3 Point-by-point optimisation . . . . . . . . . . M25

5.3 .1 Bézier curves . . . . . . . . . . . . M26

6 Conclusion

M27

\section{Introduction}

We are interested in determining the trajectory of a projectile when it is shot from a gun or artillery. The trajectory of such a projectile is affected by gravity and drag forces. There can also be transverse forces and torque but, 
for simplicity, we are interested in axisymmetric projectiles and therefore do not consider them. To state the mathematical model, we define $\mathbf{u}=(u, v, w)$ to be the projectile velocity vector, $\boldsymbol{x}=(x, y, z)$ to be the projectile position vector where $z$ is in the vertical direction, $m$ to be mass of the projectile, $t$ to be the time, $\mathbf{F}_{g}$ to be the gravitational force, and $\boldsymbol{F}_{\mathrm{d}}$ to be the drag force. The governing equations are therefore

$$
\mathrm{m} \frac{\mathrm{d} \mathbf{u}}{\mathrm{dt}}=\mathrm{F}_{\mathrm{g}}+\mathrm{F}_{\mathrm{d}}, \quad \frac{\mathrm{d} \boldsymbol{x}}{\mathrm{dt}}=\mathbf{u} .
$$

The gravitational force is straightforward and the focus of this project is to determine the drag force. In particular, data exists in several forms that documents the flight of the projectile in different scenarios, including different initial velocities and initial angles to the vertical. The main aim of this project is to determine the drag force from this data and hence enable predictions to be accurately made in other circumstances.

The drag force exerted on a projectile is determined by the flow of air around it. For simplicity we assume that the air is stationary and that the projectile moves parallel to its axis of symmetry. Hence the drag is a function of the velocity of the projectile. The drag force acts in the direction opposite to the velocity. Hence $F_{d}$ must be of the form

$$
\mathrm{F}_{\mathrm{d}}=-\mathrm{K} \frac{\mathbf{u}}{|\mathbf{u}|},
$$

where $\mathrm{K}$ is a scalar quantity that depends on physical parameters, and typically also depend on $|\mathbf{u}|$. This is consistent with the well-known drag equation,

$$
\mathrm{F}_{\mathrm{d}}=-\frac{1}{2} A \rho_{\mathrm{a}} \mathrm{C}_{\mathrm{d}} \mathbf{u}|\mathbf{u}|,
$$

where $A$ is the cross-sectional area of the projectile viewed from the the front and $\rho_{a}$ is the density of the air. The drag coefficient, $C_{d}$, may be dependent on the velocity via the dimensionless Reynold's number,

$$
\operatorname{Re}=\frac{\mathrm{L}|\mathbf{u}|}{v},
$$


where $\mathrm{L}$ is the length of the projectile and $v$ is the kinematic viscosity of the air. At subsonic speeds and low Reynolds numbers, the air flow remains laminar until separation and $C_{d}$ is inversely proportional to $R e$, so the drag force is proportional to $|\mathbf{u}|$ [3]. At subsonic speeds and high Reynolds numbers, turbulent flow is seen within boundary layers and the wake. The drag coefficient becomes approximately independent of Re, and the drag force is then proportional to $|\mathbf{u}|^{2}$. For transonic and supersonic velocities, the behaviour of $C_{d}$ changes yet again, which we explore in this paper. The projectiles of interest travel with a high Reynolds number regime, so we start by noting that the subsonic drag coefficient is approximately constant.

It is the function $C_{d}$ which is the main focus of this report; particularly, how it changes with with the local speed of sound. As the projectile travels, the density of the air may change, particularly in the vertical direction, as these projectiles may reach altitudes of several kilometres, and so the local speed of sound may change.

We now explain the fluid motion around the projectile. The equations of interest are

$$
\begin{aligned}
& m \frac{d(u, v, w)}{d t}=m(0,0,-g)-\frac{1}{2} A \rho_{a} C_{d} \sqrt{u^{2}+v^{2}+w^{2}}(u, v, w), \\
& \frac{d(x, y, z)}{d t}=(u, v, w) .
\end{aligned}
$$

Without loss of generality, the problem is simplified to motion in a single vertical plane and hence we consider just the motion in the $(x, z)$-plane and neglect the $\mathrm{y}$ behaviour.

The drag function $C_{d}$ depends on the detailed geometry of the projectile, as well as how fast the projectile is travelling through the air. Crucially, the high speed of the projectiles is such that compressible effects in the air are important. Taking the air locally to the projectile to have a speed of sound c, which depends on $z$ because both pressure and density of the air change with height, $C_{d}$ is determined by the local Mach number, defined as

$$
M=|\mathbf{u}| / \mathrm{c}(z) .
$$


Figure 1: An experimentally derived $C_{d}$ profile as a function of the Mach number, $\mathrm{M}$, for the .50 calibre ball M33 projectile [7].

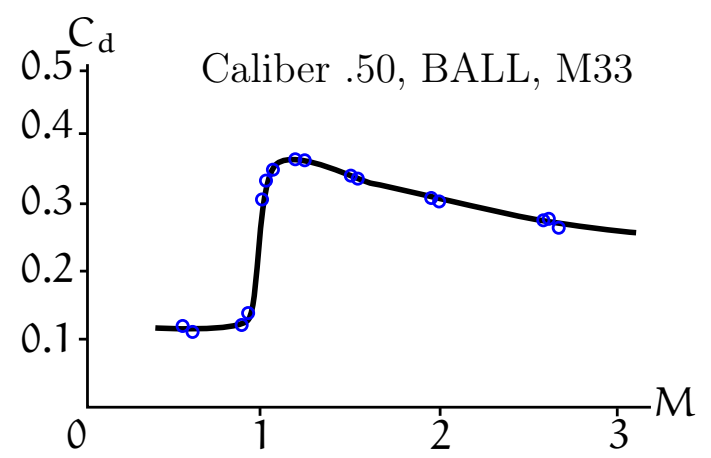

For any projectile it is possible to determine $C_{d}(M)$ from three main sources:

1. wind tunnel measurements in various conditions,

2. calculation of the fluid dynamic behaviour around the projectile and

3. using projectile path data to infer the drag coefficient.

Figure 1 shows a typical $C_{d}(M)$ profile (from experimental data [7]) which shows that $C_{d}$ is constant for subsonic speeds, has a sharp transonic rise, and then decreasing for modest supersonic speeds $\left(C_{d} \propto 1 / M\right)$. McCoy (2012) published a collection of drag profiles for many different projectiles [6]. Because source 1 is not available for many projectiles of interest, we consider some ideas that come from source 2 but direct most of our attention to source 3. The main aim of this work is to use data about the projectile path in order to extract the function $C_{d}(M)$. To assist this, we assume that the properties of the air were well-known (pressure, density and hence speed of sound, as functions of height).

\section{The physics of steady aerodynamics}

There is extensive literature on using the flow of air around objects to calculate the resulting forces [2, e.g.]. For the projectiles here we are interested in the 
steady flow of air over axisymmetric objects and concentrate on determining the drag that is induced. The main mathematical tools exploited in order to understand the behaviour are those related to slender projectiles. In all cases of interest the Reynolds number is very large and hence, away from boundary layers, we consider a compressible inviscid fluid, although there are some issues related to separation that are sensitive to viscous behaviour. The flow is taken to be quasi-static where the air properties around the projectile are assumed to be uniform but may vary slowly with time. The convention is to consider a coordinate system moving at the steady speed of the projectile and hence, in addition to the geometry of the projectile the main parameter of interest is the Mach number of the oncoming undisturbed flow. The general behaviour of the flow is then crucially dependent on whether the Mach number, $M$, is less than unity (subsonic), greater than unity (supersonic) or close to unity (transonic). There is a body of literature for the background theory [5, 8, 4] and computational fluid dynamics [1].

For the remainder of the chapter, we examine each of the different regimes of the flow behaviour and only consider the case of a slender projectile so the flow equations are simplified.

\subsection{Subsonic flow $(M<1)$}

The velocity of air is described by the wave equation,

$$
\nabla^{2} \phi=\frac{1}{c^{2}} \frac{\partial^{2} \phi}{\partial t^{2}},
$$

where $\mathbf{u}=\nabla \phi$. This arises from the linearisation of the governing Euler, continuity and energy equations of an ideal gas [8]. For a moving coordinate system travelling at the projectile speed, $\mathrm{U}$, the resulting modified wave PDE $[8,9]$ is

$$
\nabla^{2} \phi=\frac{1}{c^{2}}\left(\frac{\partial}{\partial t}+\mathrm{u} \frac{\partial}{\partial x}\right)^{2} \phi .
$$


Figure 2: Approximate flow field (dotted region is the wake) and the distribution of the pressure coefficient for subsonic flow.
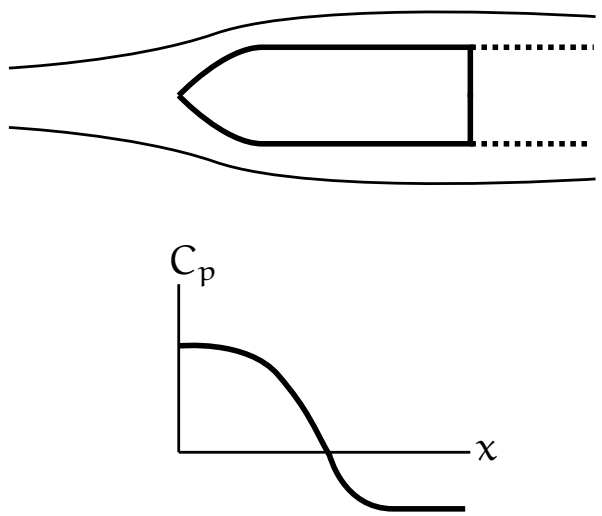

For steady flow over an axisymmetric particle, the governing PDE of interest is then

$$
\left(1-M^{2}\right) \frac{\partial^{2} \phi}{\partial x^{2}}+\frac{1}{r} \frac{\partial}{\partial r}\left(r \frac{\partial \phi}{\partial r}\right)=0,
$$

where $\mathrm{M}=\mathrm{U} / \mathrm{c}$.

At subsonic speeds, $M<1$ so (10) is elliptic. Therefore the flow is affected everywhere by the shape of the projectile. A simple rescaling of lengths then dictates the behaviour is governed by Laplace's equation. Central to the behaviour is the boundary layer which separates from the projectile at some point (if it does not, then the drag on the projectile may become zero - called D'Alembert's paradox). The base of the projectile has a sharp rim which ensures that separation occurs. In the separated region (the "wake") the pressure will be nearly constant and needs to be determined separately.

Figure 2 shows the basic flow and that the main contribution to the drag is a high pressure region near the nose of the projectile and a low pressure at the rear due to separation and the wake. An approximate formula for the drag is

$$
\mathrm{C}_{\mathrm{d}} \propto \int(\mathrm{p} \times \text { surface slope }) \mathrm{d} S \approx \text { constant; }
$$


the pressure coefficient is

$$
C_{p}=\frac{p-p_{\infty}}{\rho_{\infty} U^{2} / 2}
$$

where $p$ is the pressure at the point, $p_{\infty}$ is the pressure in the oncoming freestream, $\rho_{\infty}$ is the freestream fluid density, and $\mathrm{U}_{\infty}$ is the freestream velocity of the fluid [9]. In practice it is found that $C_{d}$ slowly varies with Mach number in this regime, as seen in many profiles [6].

The assumptions used to derive the PDE (10) no longer apply as $M \rightarrow 1$. In this limit, the coefficient of the $x$ derivative becomes small and discarded nonlinear terms become important to maintain the energy balance. Thus, to explain transonic behaviour, where $M \rightarrow 1$, more care is needed, as we show in Section 2.3.

\subsection{Supersonic flow $(M>1)$}

If the projectile is travelling fast enough, then the oncoming flow is supersonic and the velocity potential $\phi$ satisfies the linear PDE

$$
\mathrm{B}^{2} \frac{\partial^{2} \phi}{\partial x^{2}}-\frac{1}{r} \frac{\partial}{\partial r}\left(r \frac{\partial \phi}{\partial r}\right)=0
$$

where $B=\sqrt{M^{2}-1}$. This PDE differs from the subsonic linear PDE (10) because the coefficient of the $x$ derivative has changed sign. Thus, (13) is hyperbolic and therefore there are characteristics along which information travels from the projectile outwards. The main properties of the flow are shown in Figure 3 indicating that a shock wave forms at both the front and rear of the projectile.

Figure 3 also shows the distribution of the pressure coefficient along the surface of the projectile and this is dominated by the large pressure peak at the front of the projectile just behind the shock wave. In 2D flow, and where 
Figure 3: Supersonic flow over a projectile showing the shock wave structure and the resulting distribution of the pressure coefficient.

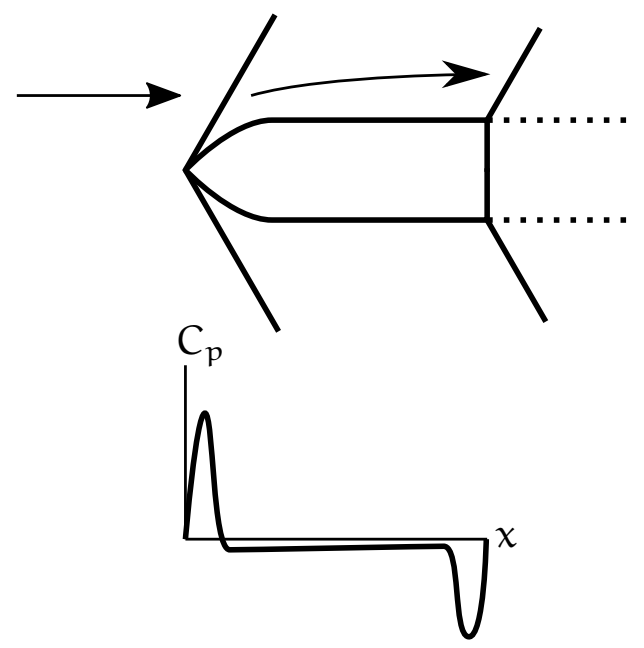

$r=f(x)$ describes the surface profile, the drag coefficient scales like

$$
C_{d} \propto \frac{f^{\prime}(x)}{B} \sim \frac{f^{\prime}(x)}{M} .
$$

As with the subsonic case, the linear supersonic model breaks down as $M \rightarrow 1$. A nonlinear model is required to capture the transonic behaviour near $M \approx 1$.

\subsection{Transonic flow $(M \approx 1)$}

The transonic case is much more complicated, particularly as the behaviour cannot be linearised. In this case the velocity potential satisfies

$$
\left(K_{1}\left(1-M^{2}\right)-K_{2} \frac{\partial \phi}{\partial x}\right) \frac{\partial^{2} \phi}{\partial x^{2}}-\frac{1}{r} \frac{\partial^{2}}{\partial r}\left(\frac{\partial \phi}{\partial r}\right)=0,
$$

where $K_{1}$ and $K_{2}$ are constants which may depend on the Mach number, speed of sound, and the heat capacity ratio of the air $[8,9]$. 
Figure 4: Flow field and pressure coefficient for the case where the supersonic bubble has just formed $(M=1-\mathcal{O}(\epsilon))$.
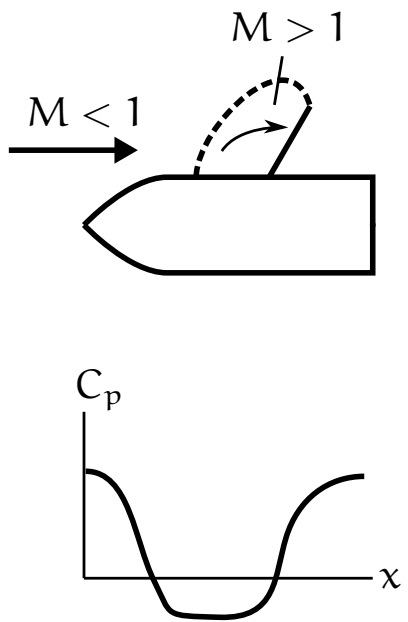

We see from PDE (15) that the behaviour may be elliptic in some regions and hyberbolic in others, depending on the value of $\partial \phi / \partial x$. Thus, the equation is of a mixed type. For example, when $M=1$, PDE (15) is hyperbolic or elliptic depending on the sign of $\partial \phi / \partial x$.

To examine transonic flow, PDE (15) is considered in the limit where $M=1-\epsilon$ with $0 \leqslant \epsilon \ll 1$. The resulting behaviour consists of the different regions which are typically as shown in Figure 4 where the problem is subsonic in some parts where the governing equation is elliptic, and supersonic in other parts where the PDE is hyperbolic. The transition from subsonic to supersonic is relatively smooth but the reverse transition causes a shock to form. A "bubble" of supersonic flow therefore forms on the projectile and this region grows as the Mach number approaches unity. When the shock at the rear of this bubble reaches the base of the projectile there is the possibility that the resulting pressure changes may cause a reduction of the drag coefficient. There is some very interesting behaviour as the Mach number reaches and increases past unity. The bubble grows and extends to the full length of the projectile as well as extending radially to infinity. There is then a complex structure of characteristics within the bubble with part of the bubble affected by the upstream flow and the rear part of the bubble affected by the flow 
Figure 5: Flow field and pressure coefficient for the case where the supersonic bubble has grown to infinity $(M=1-\mathcal{O}(\delta)$, where $\delta<\epsilon)$. Decreasing from $M>1$ to $M<1$ gives quite a different picture, where no supersonic bubble is present.

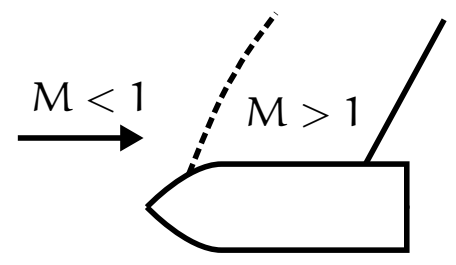

as $r \rightarrow \infty$. Separating these two regions is a "limiting characteristic" [5].

The basic structure of the pressure coefficient for the case of when the projectile first creates a bubble and the later behaviour when the bubble extends to infinity are shown in Figures 4 and 5. Transonic behaviour for $M>1$ is shown in Figure 6. The main features are the very large pressure at the front of the projectile as the flow meets the projectile and creates a shock wave. Some of these flow figures are unstable configurations, and depend on whether the projectile approaches transonic speeds from above or below. Thus, they should only be used for illustrative purposes.

\section{Low angle approximations to the projectile path}

For many projectiles the path is relatively straight because the angle to the horizontal is very small and hence the path rises slightly above the surface of the ground before falling and hitting the surface. The path is therefore dominated by the horizontal speed of the projectile. We exploit this small 
Figure 6: Flow field and pressure coefficient for slightly supersonic speeds in the transonic regime $(M=1+\mathcal{O}(\epsilon))$. A bubble of subsonic flow persists near the nose.

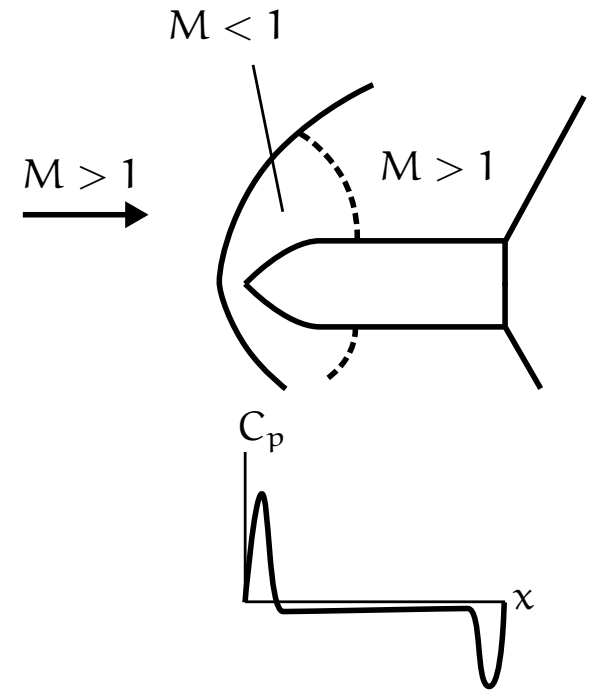

rise in order to simplify the path and hence identify methods to extract the drag coefficient. This also emphasises some of the difficulties that occur when considering more general projectile paths.

For this section and the next we consider the local sound speed to be $\mathrm{c}$ and nondimensionalise the problem using

$$
\mathrm{u}=\mathrm{c} \hat{u}, \quad w=c \hat{w}, \quad t=\frac{2 m}{A \rho_{\mathrm{a}} \mathrm{c}} \hat{\mathrm{t}}, \quad x=\frac{2 \mathrm{~m}}{A \rho_{\mathrm{a}}} \hat{x}, \quad z=\frac{2 \mathrm{~m}}{A \rho_{\mathrm{a}}} \hat{z} .
$$

Omitting the "hat" notation for simplicity,

$$
\frac{d(u, w)}{d t}=(0,-J)-C_{d} \sqrt{u^{2}+w^{2}}(u, w), \quad \frac{d(x, z)}{d t}=(u, w),
$$

where the Mach number is $\sqrt{u^{2}+w^{2}}$ and $C_{d}$ depends on this value only, and there is one dimensionless parameter

$$
J=\frac{2 m g}{A \rho_{\mathrm{a}} c^{2}}
$$


which represents the ratio of the gravitational force to the drag near sonic speeds. The density of any projectile is approximately constant with $\rho_{\text {proj }} \approx$ $3 \times 10^{3} \mathrm{~kg} / \mathrm{m}^{3}$, and since the mass is approximately $\mathrm{m}=\rho_{\text {proj }} A L$ we find that

$$
\mathrm{J} \approx \frac{\rho_{\text {proj }}}{\rho_{\mathrm{a}}} \frac{2 \mathrm{~g}}{\mathrm{c}^{2}} \mathrm{~L},
$$

and hence this varies from projectile to projectile due only to differences in length.

For low trajectory projectiles we know that $z$ and $w$ are small so we scale them with the initial angle to the horizontal $\theta$ so that the system becomes

$$
\begin{aligned}
& \frac{d u}{d t}=-C_{d} \sqrt{u^{2}+\theta^{2} w^{2}} u, \quad \frac{d w}{d t}=-J / \theta-C_{d} \sqrt{u^{2}+\theta^{2} w^{2}} w, \\
& \frac{d(x, z)}{d t}=(u, w),
\end{aligned}
$$

with initial data $u=M_{0} \cos (\theta), w=M_{0} \sin (\theta) / \theta, x=0, z=0$, where $M_{0}$ is the initial Mach number of the projectile.

Asymptotic analysis could be done using $\theta \rightarrow 0$ and various cases for $\mathrm{J}$ but we present just one example. If $J / \theta \ll 1$ then, the lowest order problem is

$$
\frac{\mathrm{du}}{\mathrm{dt}}=-\mathrm{C}_{\mathrm{d}}|\mathrm{u}| \mathrm{u}, \quad \frac{\mathrm{d} w}{\mathrm{dt}}=-\mathrm{J} / \theta, \quad \frac{\mathrm{d}(x, z)}{\mathrm{dt}}=(\mathrm{u}, w),
$$

with $u=M_{0}, w=M_{0}, x=0, z=0$ at $t=0$.

Noting that the local Mach number $M \approx|u|$ so $C_{d}$ only depends on $\mathfrak{u}$, the equation for $u$ now decouples from the system, and assuming $M_{0} \geqslant u$ and $u>0$,

$$
\int_{u}^{M_{0}} \frac{d u}{u^{2} C_{d}}=t
$$

Similarly we find

$$
z=M_{0} t-\frac{J}{2 \theta} t^{2}, \quad \text { and } \quad \int_{u}^{M_{0}} \frac{d u}{u C_{d}}=x .
$$


The simple expressions in (24) are now used to find approximations for $C_{d}$ by fitting the results to the data. For example, consider multiple trials, at a constant $M_{0}$ with different launch angles giving measured data $t_{f}$ and $u_{f}$, where $\boldsymbol{t}_{\boldsymbol{f}}$ is the time of flight and $\mathfrak{u}_{\boldsymbol{f}}$ is the final speed. Labelling each of these trials by index $x$,

$$
\int_{u_{f}(x)}^{M_{0}(x)} \frac{d u}{C_{d}(u) u^{2}}=t_{f}(x) .
$$

Differentiating (25) with respect to $x$ gives

$$
C_{d}\left(u_{f}\right)=-\frac{1}{u_{f}^{2}} \frac{d u_{f}}{d x} \frac{1}{d t_{f} / d x} .
$$

We simply make numerical approximations of the derivatives in (26) from the data to find the value of $C_{d}\left(u_{f}\right)$. An example of the results found from such a procedure is given in Figure 7. One important observation to make, is that it is not possible to infer behaviour of $C_{d}$ for values of the Mach number that do not occur during the experiments that produce the data. This is particularly problematic for the nearly horizontal shots of this regime; the projectiles have a short time of flight and tend to remain at supersonic speeds from firing to impact. Thus, experimental data is quite sparse for horizontal shots spanning transonic velocities.

\section{Attraction to Mach 1}

Numerical studies indicate that many projectiles appear to travel at Mach 1 for a considerable distance even when their initial speed was much higher. This provides a broad experimental window in which to study the behaviour of transonic projectiles.

We now examine the flight of a projectile in the case of a highly simplified drag coefficient to understand this behaviour. We neglect any variations with 
Figure 7: The drag coefficient, $C_{d}$, calculated using numerical differentiation for a low angle projectile.

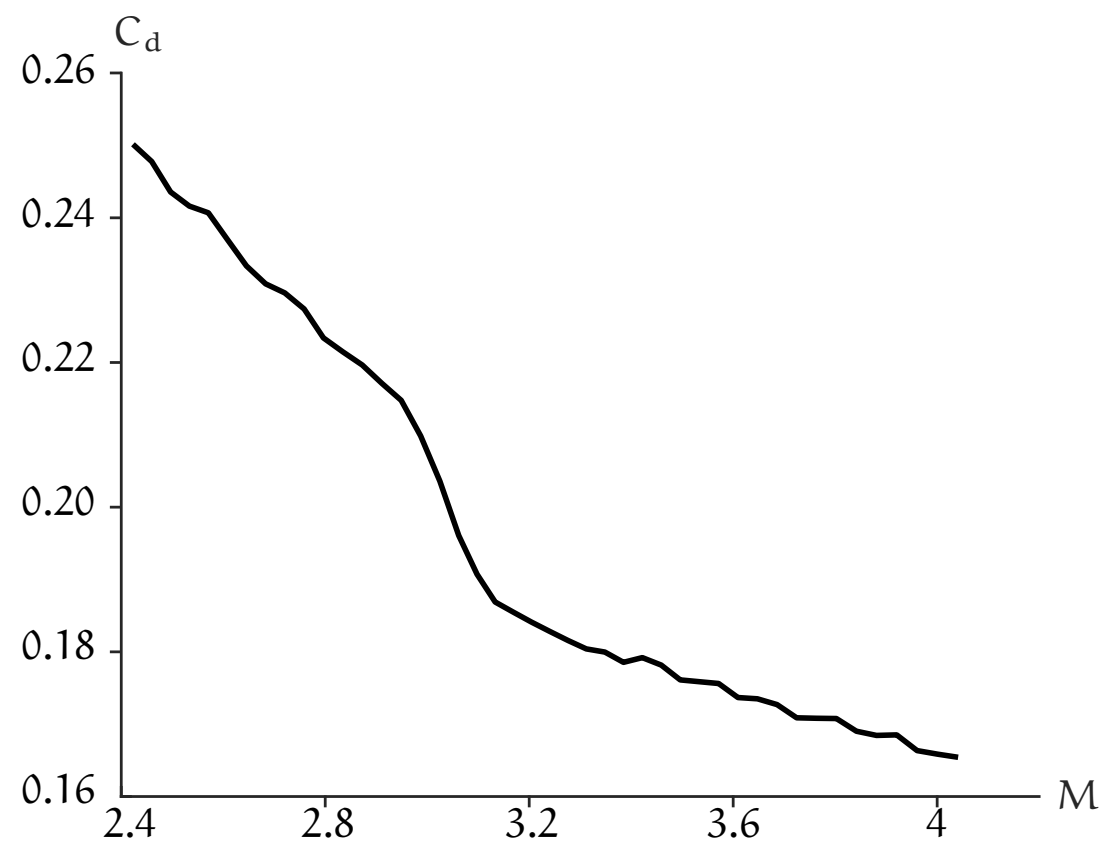

height of the density or speed of sound and analyse the problem in the phase plane to identify generic behaviour.

The simplest case to consider is a projectile that is shot vertically upwards, reaches a maximum height, turns to face downwards and returns to earth. We might expect that the projectile reaches a terminal velocity on its return if it is shot up at sufficiently high velocity. We start by studying what that terminal velocity might be. With just vertical motion the dimensionless equations are

$$
\frac{\mathrm{d} w}{\mathrm{dt}}=-\mathrm{J}-\mathrm{C}_{\mathrm{d}}|w| w, \quad \frac{\mathrm{d} z}{\mathrm{dt}}=w,
$$

where the local Mach number is now $|w|$ and $C_{d}$ depends only on this. The 
terminal velocity occurs when the speed on the descent $(w<0)$ is such that

$$
\mathrm{J}=\mathrm{C}_{\mathrm{d}} w^{2} .
$$

In almost all practical cases there is a single value of $w$ where (28) occurs and it is instructive to examine this further. We consider a very simple form for the drag coefficient where it is a step function shape rising sharply from constant $\mathrm{a}$ to constant $\mathrm{b}$ across Mach $1(|w|=1)$. For such a drag coefficient there are three different types of behaviour, determined by the values of $\mathrm{J}$, a and $b$ :

- for $\mathrm{J}<\mathrm{a}$, the terminal velocity is $w=\sqrt{\mathrm{J} / \mathrm{a}}$ (subsonic),

- for $\mathrm{a}<\mathrm{J}<\mathrm{b}$, the terminal velocity is $w=1$ (sonic),

- for $\mathrm{J}>\mathrm{b}$, the terminal velocity is $w=\sqrt{\mathrm{J} / \mathrm{b}}$ (supersonic).

Hence, the terminal velocity can be subsonic or supersonic but, because of the rapid variation in drag coefficient at the sonic point, there are a range of projectiles whose terminal velocity is at the speed of sound (and many projectiles, in practice, lie in this region).

To examine this more closely we consider the path of a projectile in a plane where the equations for the velocity are

$$
\frac{d(u, w)}{d t}=(0,-J)-C_{d} \sqrt{u^{2}+w^{2}}(u, w),
$$

and $C_{d}$ depends only on $\sqrt{u^{2}+w^{2}}$, that is, we ignore the variation of air parameters with height and assume the simple $\mathrm{a}, \mathrm{b}$ step function behaviour. We now consider the phase plane $(\boldsymbol{u}, \boldsymbol{v})$ and (29) becomes

$$
\frac{d w}{d u}=\frac{-J-C_{d} \sqrt{u^{2}+w^{2}} w}{-C_{d} \sqrt{u^{2}+w^{2}} u}
$$

and the equilibrium points of this system occur when $u=0$ and $J=-C_{d}|w| w$ (which are exactly the cases described for the one-dimensional case). However, 
Table 1: Stable regions for different regimes.

\begin{tabular}{ccc}
\hline $\mathrm{J}$ value & Terminal velocity & Stable region on $w=-\sqrt{1-\mathrm{u}^{2}}$ \\
\hline $\mathrm{J}<\mathrm{a}$ & subsonic & none \\
$\mathrm{a}<\mathrm{J}<\mathrm{b}$ & sonic & $0<\mathrm{u}<\sqrt{1-\frac{\mathrm{a}^{2}}{\mathrm{~J}^{2}}}$ \\
$\mathrm{~J}>\mathrm{b}$ & supersonic & $\sqrt{1-\frac{\mathrm{b}^{2}}{\mathrm{~J}^{2}}}<\mathrm{u}<\sqrt{1-\frac{\mathrm{a}^{2}}{\mathrm{~J}^{2}}}$ \\
\hline
\end{tabular}

the equations also have some very interesting behaviour because there are cases where the projectile remains at the sonic speed. To explain these cases, we assume the projectile is at the sonic point $u^{2}+w^{2}=1$ and ask what values of $u$ ensure that we remain on this sonic curve. Putting this assumption into (30) we find the projectile remains on the sonic curve if $J \sqrt{1-u^{2}}=C_{d}$. Again we can now consider the step function shape where $C_{d}$ can take any value between and including $\mathbf{a}$ and $\boldsymbol{b}$. This identifies the region of $\boldsymbol{u}$ (Table 1 ) where solutions that arrive on the curve $w=-\sqrt{1-u^{2}}$ remain on the curve (we call these stable regions).

To illustrate these different behaviours a phase plane, showing the direction field (as arrows) and several possible solution paths, is given for each of the three cases. A collection of example trajectories are shown for subsonic terminal velocities or $\mathrm{J}<\mathrm{a}$ (Figure 8), transonic terminal velocities or $\mathrm{a}<\mathrm{J}<\mathrm{b}$ (Figure 9) and supersonic terminal velocities or $\mathrm{J}>\mathrm{b}$ (Figure 10). For transonic terminal velocities, a wide range of initial conditions lead to trajectories that remain on the sonic line before coming to rest at terminal velocity. This behaviour extends to supersonic terminal velocities, where trajectories spend a lot of time near the sonic line before diverting to the terminal velocity.

In practice the drag coefficient does not have a jump at the sonic speed but it does have a significant step over a small Mach number range. If we can estimate this jump, then these phase planes allow an important observation to be made. If the projectile has an estimated value of $J$ that makes its terminal velocity subsonic, then it will be very difficult to explore the behaviour of $C_{d}$ 
Figure 8: Velocity $(u, w)$ phase plane with $\mathrm{J}<\mathrm{a}$ so the terminal velocity is subsonic. The green line denotes the sonic curve (Mach 1). Trajectories easily cross the Mach 1 line to reach terminal velocity (red dot).

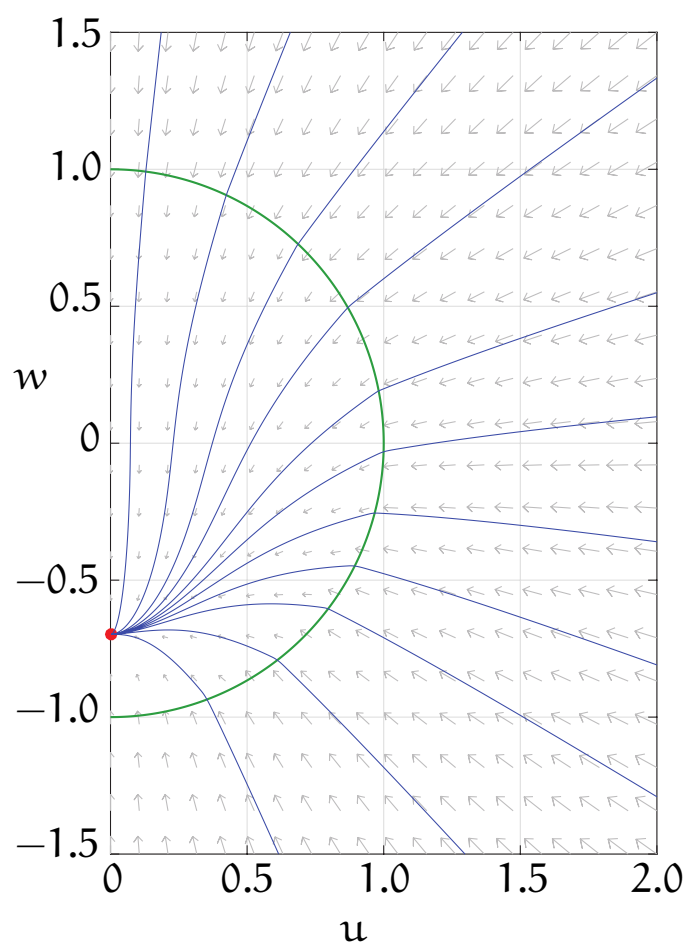

near the sonic point because any path will stay near there for only a short time. However, for other values of $J$ there are projectile paths that spend significant time near the sonic speed and if these paths are included in the data, then it should be possible to extract details of what $C_{d}$ does in this region. In reality, the practical issues, such as the Mach number depending on the height of the projectile through variations in the sound speed, make some of these suggestions difficult to implement in practice, as discussed in Section 5.2. 
Figure 9: Velocity $(u, w)$ phase plane with $\mathrm{a}<\mathrm{J}<\mathrm{b}$ so the terminal velocity is transonic. The green line denotes the sonic curve. Trajectories tend to reach the sonic curve and remain on it until they reach the transonic terminal velocity (red dot).

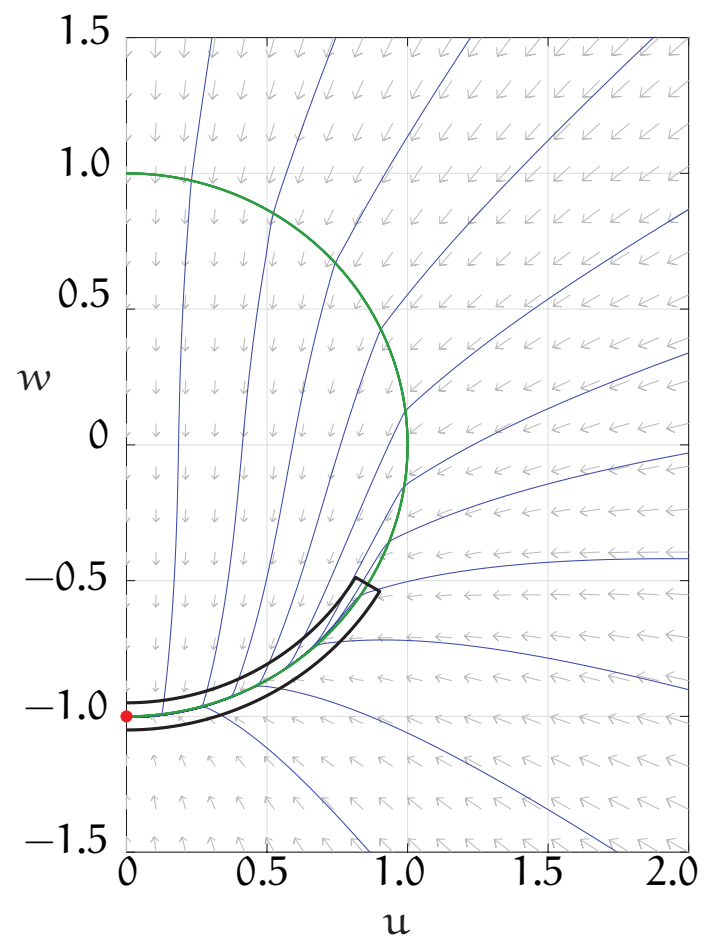

\section{Possible approximations to the drag coefficient}

From the existing large data sets of drag on projectiles and the associated aerodynamics, we now consider what possible approximations to the drag coefficient can be made that enables simple optimisation of the predicted paths to the tabular data from measured paths to be performed easily. One of the main issues is the number of parameters needed to describe the drag coefficient. 
Figure 10: Velocity $(\mathfrak{u}, \mathfrak{w})$ phase plane with $\mathrm{J}>\mathrm{b}$ so the terminal velocity is supersonic. The green line denotes the sonic curve. Trajectories tend to reach the sonic curve and remain on it for a while before diverting to the supersonic terminal velocity (red dot).

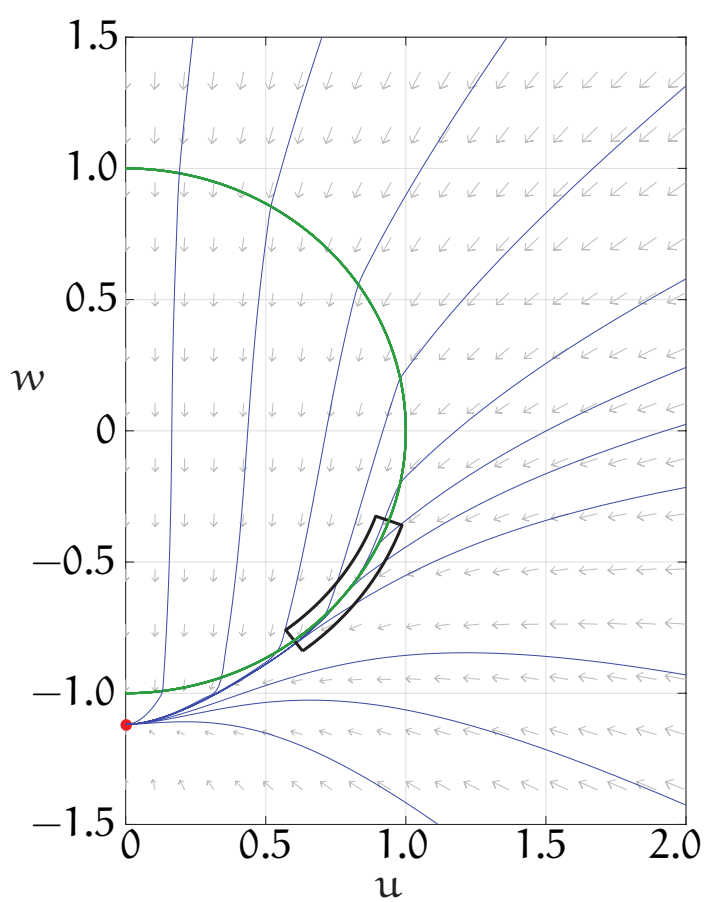

\subsection{Functional forms for $C_{d}$}

For the drag coefficient, researchers at the Defence Science and Technology group often employ a family of exponential-type smoothed step functions with seven free parameters. The optimal drag profile is then found by comparison with firing table data. The functional form is

$$
\begin{aligned}
& C_{d}=\alpha+\frac{\beta}{M}, \quad \text { where } \\
& \alpha=\alpha_{0}+\frac{\alpha_{1}}{1+\exp \left[-w_{\alpha}^{-1}\left(M-\alpha_{\mu}\right)\right]}, \\
& \beta=\frac{\beta_{1}}{1+\exp \left[-w_{\beta}^{-1}\left(M-\beta_{\mu}\right)\right]} .
\end{aligned}
$$


Figure 11: An example drag coefficient profile for the three-parameter family, for $a=0.25, b=0.1$, and $c=10$.

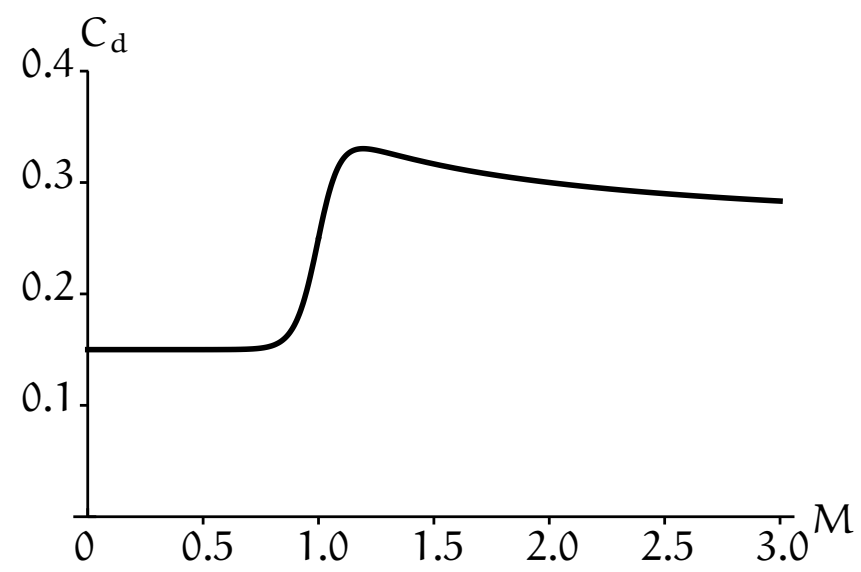

The seven free parameters are $\alpha_{0}, \alpha_{1}, w_{\alpha}, \alpha_{\mu}, \beta_{1}, w_{\beta}$ and $\beta_{\mu}$. To reduce the number of free parameters, we proposed a three-parameter family of piecewise defined hyperbolic tangent functions. These functions are

$$
C_{d}=\left\{\begin{array}{lc}
a+b \tanh [c(M-1)], & M \leqslant 1, \\
a+\frac{b}{M} \tanh [c(M-1)], & M>1 .
\end{array}\right.
$$

An example drag coefficient profile using (34) is shown in Figure 11 which clearly captures the experimental profile shown in Figure 1.

The drag coefficient profile of some projectiles cannot be represented by this three-parameter family. For example, experiments show that some projectiles exhibit a characteristic "dip" where $C_{d}$ drops slightly before the transonic rise. For such projectiles, the following five-parameter family of functions 
Figure 12: An example drag coefficient profile from the five-parameter family, for $\mathrm{a}=0.25, \mathrm{~b}=0.1, \mathrm{c}=10, \mathrm{~d}=5$, and $e=50$.

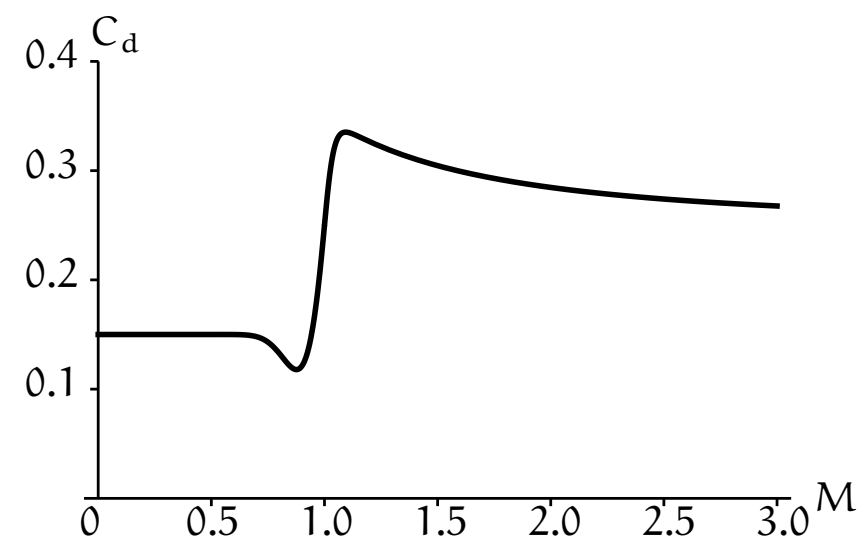

may be more appropriate:

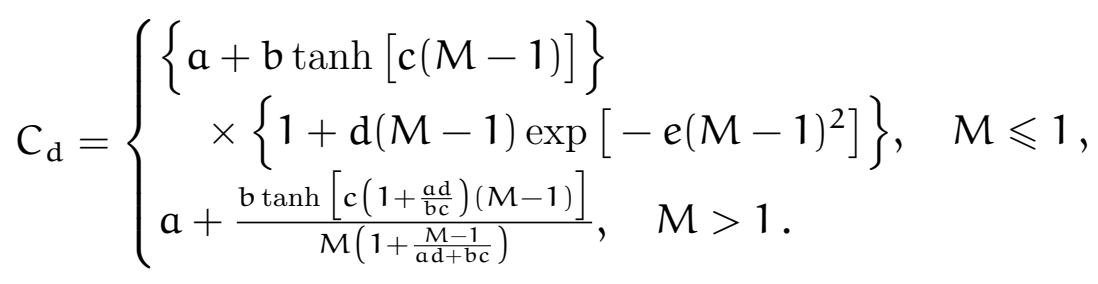

Figure 12 shows an example of a five-parameter profile using (35).

\subsection{Optimising with the three-parameter hyperbolic tangent}

We now proceed to use the three-parameter family to optimise the values for $\mathbf{a}, \boldsymbol{b}$ and $\mathbf{c}$ against experimental firing tables (Table 2). The firing tables are considered a guide for marksmen who need to determine the angle of fire given the distance to target (and a consistent muzzle velocity). This is why the range appears to be the independent variable spread $100 \mathrm{~m}$ apart. Our 
Table 2: Experimental firing table data from left to right: distance to ground impact, angle of fire (independent variable), angle of impact, peak height, horizontal distance of peak height, flight time, final velocity.

\begin{tabular}{rlllrcc}
\hline$R$ & Angle & Fall & topH & topR & Time & Vf \\
\hline 100 & 0.0833 & 0.0667 & 0.03 & 51 & 0.15 & 623 \\
300 & 0.2333 & 0.3000 & 0.34 & 162 & 0.52 & 459 \\
500 & 0.4833 & 0.8000 & 1.3 & 282 & 1.04 & 334 \\
600 & 0.6667 & 1.1500 & 2.3 & 344 & 1.35 & 304 \\
800 & 1.1000 & 2.1000 & 5.5 & 468 & 2.05 & 266 \\
\hline
\end{tabular}

calculations take the form of initial value problems where the firing angle and muzzle velocity are independent variables, while the range is used as a target metric. The optimisation proceeds as follows:

1. For a given firing angle, calculate a trajectory for an initial $a, b$ and $c$.

2. Compare trajectory values (e.g., velocity of impact, peak height) with experimental data

3. modify $a, b$ and $c$ to improve the result.

4. repeat.

We used this optimisation procedure for each angle on the available firing table data (including Table 2). The resulting parameters for each angle are shown in Table 3, with the corresponding drag profiles shown in Figure 13. The $r$ values are the ratio between the tabulated data and the simulated flight using optimised parameters, with $r=1$ being a perfect fit.

There are a few issues with the data in Table 2. For any given projectile, we would expect that the calculated drag coefficient profile is consistent for any given firing angle, which is not the case. To remedy this, the optimisation 
Figure 13: Drag coefficient profiles optimised for each of the eight firing table angles in Table 3.

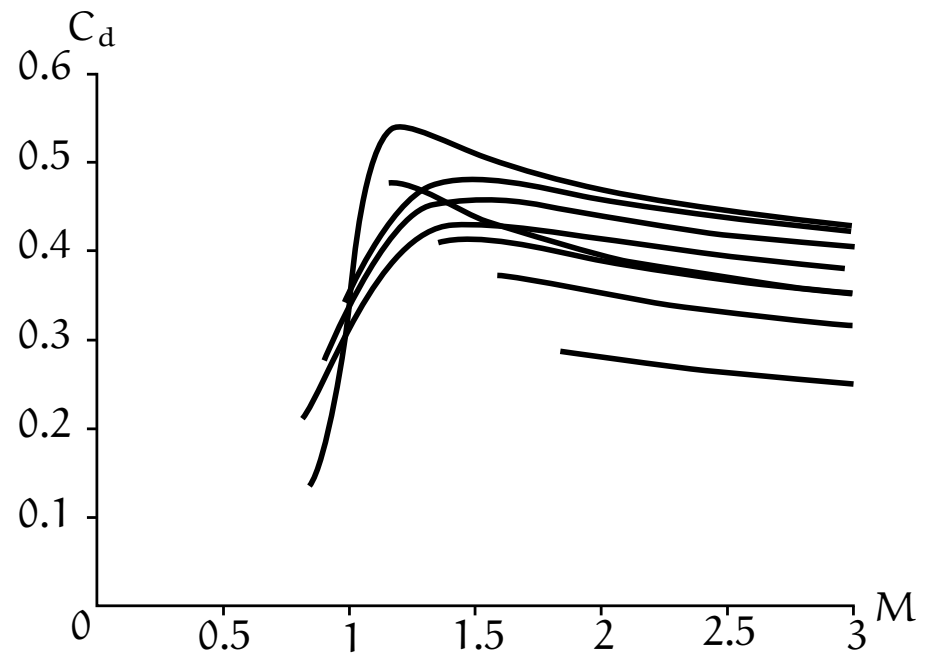

Table 3: Parameter and $\boldsymbol{r}$ values for each angle

\begin{tabular}{llcccccccc}
\hline$\theta$ & $r(\mathrm{R})$ & $\mathrm{r}(\theta)$ & $\mathrm{r}(\mathrm{Vf})$ & $\mathrm{r}(\mathrm{ToF})$ & $\mathrm{r}(\mathrm{TopH})$ & $\mathrm{r}(\mathrm{TopR})$ & $\mathrm{a}$ & $\mathrm{b}$ & $\mathrm{c}$ \\
\hline 0.08 & 1.38 & 1 & 1.00 & 1.38 & 1.73 & 1.38 & 0.19 & 0.18 & 2.9 \\
0.15 & 1.119 & 1 & 1.00 & 1.13 & 1.23 & 1.11 & 0.24 & 0.22 & 3 \\
0.23 & 1.03 & 1 & 1.00 & 1.04 & 1.07 & 1.02 & 0.27 & 0.23 & 3.1 \\
0.33 & 0.991 & 1 & 0.99 & 0.99 & 0.98 & 0.98 & 0.26 & 0.26 & 11 \\
0.48 & 0.960 & 1 & 1.00 & 0.98 & 1.01 & 0.96 & 0.35 & 0.21 & 3 \\
0.66 & 0.984 & 1 & 1.00 & 0.99 & 1.00 & 0.98 & 0.33 & 0.20 & 2.9 \\
0.96 & 0.991 & 1 & 1.00 & 1.04 & 1.12 & 0.98 & 0.35 & 0.23 & 9.5 \\
1.1 & 1.00 & 1 & 1.00 & 0.99 & 0.98 & 0.98 & 0.31 & 0.21 & 2.7 \\
\hline
\end{tabular}


Figure 14: Unusual experimental radar drag profiles.
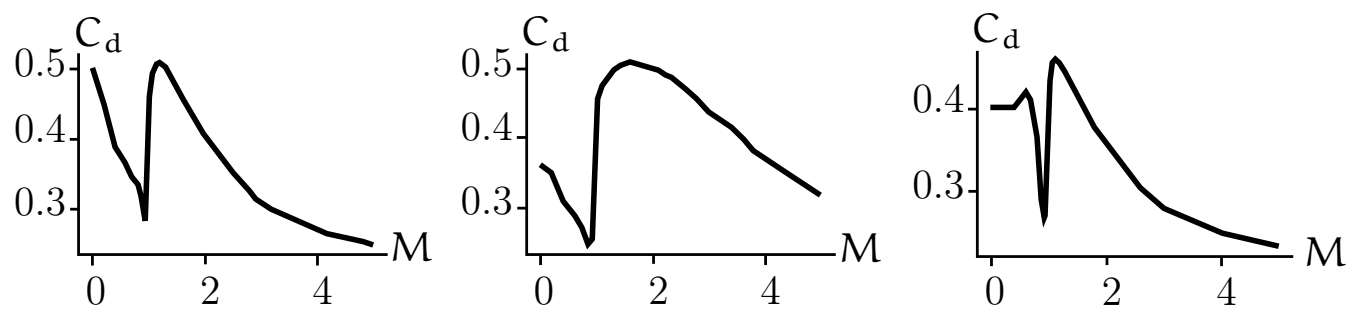

procedure should be tweaked to globally optimise against the data from all firing angles. This is complicated as each firing angle spans a different range of Mach numbers. Some sort of special weighting algorithm may be needed. Another issue highlighted is that only a few firing angles yield Mach numbers in the transonic regime which is what we are particularly interested in. Most firing angles are near-horizontal, so the projectile impacts before losing too much speed. The lack of data in this interesting regime was the inspiration behind the discussion in Section 4.

\subsection{Point-by-point optimisation}

Another avenue for obtaining the drag profile is from point-by-point optimisation. Here, the profile is not parametrised, but is discretised into $n$ points which are free to move by the optimisation procedure. The advantage is that here we:

- obtain the drag coefficient for exceptional projectiles that are not suitable parametrised by the above families; and

- identify the small details of the drag coefficient profiles.

For example, Figure 14 shows unusual experimental drag coefficient profiles. The main disadvantage is that the procedure takes more time than the parametrised functions as there are $n$ free parameters where $n \approx 40$ for 
Figure 15: Left: A noisy drag coefficient given by a piecewise linear function (dashed red) and a smooth but noisy function (solid black). Right: The equivalent profile where the high frequency noise is smoothed by Bézier curves.
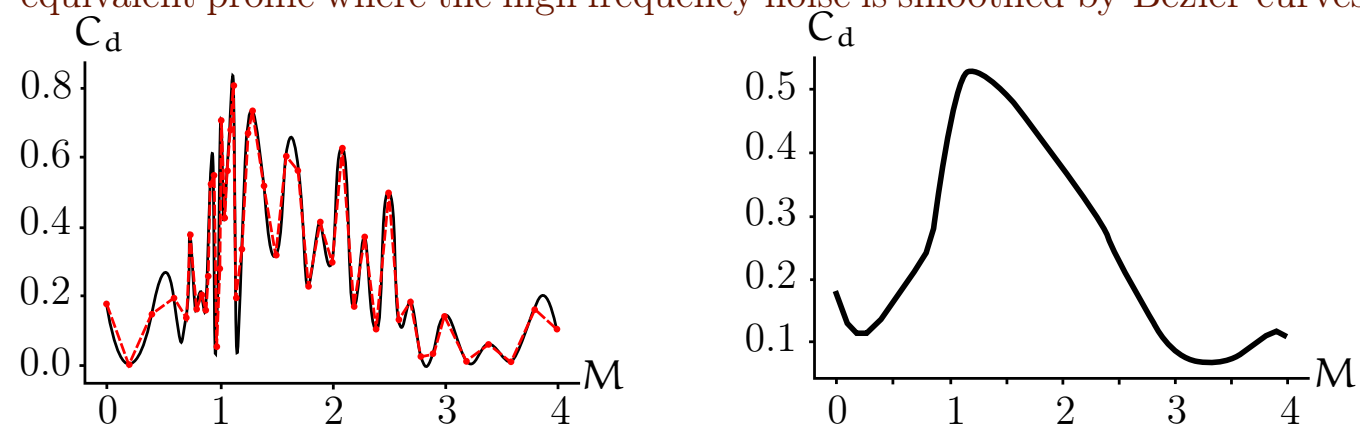

accuracy which is far greater than the three- or five-parameter families. Another disadvantage is that we lose any smooth behaviour and this makes it difficult to gain physical insight into what may be causing the changes in drag.

\subsubsection{Bézier curves}

The drag coefficient profiles obtained by point-by-point optimisation are often very noisy and rough. It is possible to smooth out these profiles by approximating the optimal results by Bézier curves, which are based on Bernstein polynomials, and are commonly used in computer graphics. A comparison between an example noisy drag profile and the smoothed version is shown in Figure 15. Ideally, this smoothed profile would then be used to recalculate the projectile trajectory to check that the target metrics are not too different from those of the optimum profile. 


\section{Conclusion}

We began our investigation by trying to gain a greater understanding of the fluid dynamics involved to help our physical intuition. The behaviour of a fast-moving projectile in air is complicated, particularly in the sonic regime, so it is often necessary to appeal to techniques like optimisation as a route to solving the inverse problem for the drag coefficient. To this end, in Section 5, we suggested alternative parametrisations and smoothing techniques for the drag coefficient profiles to improve the efficiency and utility of the optimisation procedure.

A major barrier is the lack of data, particularly for the sonic regime in which we are most interested. Using idealised smoothed step functions as a representative drag profile, we demonstrated in Section 4 that projectiles with a terminal velocity near or above sonic speeds will spend a large amount of time travelling near Mach 1. This provides an ideal experimental window in which to gather experimental data for the transonic region. Making use of terminal velocities and vertical firing experiments is particularly appealing because of this attraction to Mach 1. For the usual wind tunnel experiments where the speed is the control variable, we find that good dense data at Mach 1 is difficult to obtain as a small change in velocity lead to a large change in the drag coefficient due to the sharp transonic rise.

It may very well be beyond the scope of our industry partner to obtain the data from vertical firing experiments. More data would be beneficial to both the physical understanding of projectile flight, and the optimisation procedure used to obtain the drag coefficient profile.

Acknowledgements We thank Oleg Mazonka who brought this problem from the Defence Science and Technology group. We also thank the participants of this MISG study group for their valuable contributions: Jin-Hwan Cho, Patrick Hassard, Yuta Hatakeyama, John van der Hoek, Kaname Matsue, Sayyed Mirnaziry, Ignacio Ortega, and Bill Whiten. 


\section{References}

[1] O. O. Bendikson. "Review of unsteady transonic aerodynamics theory and applications". In: Progress in Aerospace Sciences 47 (2011), pp. 135-167. DOI: 10.1016/j.paerosci.2010.07.001 (cit. on p. M6).

[2] L. J. Clancy. Aerodynamics. Wiley, 1975 (cit. on p. M5).

[3] R. Clift, J. R. Grace, and M. E. Weber. Bubbles, drops, and particles. New York: Academic Press, 1978. DOI: 10.1017/S0022112079221290 (cit. on p. M4).

[4] A. B. Fonarev, J. T. Madhani, and M. A. Naida. "An effective method of calculating transonic flows and 3-D elongate bodies within framework of transonic equivalence rule". In: $14^{\text {th }}$ Australasian Fluid Mechanics Conference, Adelaide. 2001 (cit. on p. M6).

[5] K. G. Guderley. The theory of transonic flow. Pergamon, 1962. DOI: 10.1002/zamm. 19630430318 (cit. on pp. M6, M11).

[6] R. L. McCoy. Modern exterior ballistics. Atglen, Pennsylvania: Schiffer military history, 2012 (cit. on pp. M5, M8).

[7] R. L. McCoy. The aerodynamic Characteristics of .50 Ball, M33, API, M8, and APIT, M20 Ammunition. Tech. rep. BRL-MR-3810. Ballistic research laboratory memorandum, 1990 (cit. on p. M5).

[8] H. Ockendon and J. R. Ockendon. Waves and compressible flow. Springer, 2016. DOI: 10.1007/b97537 (cit. on pp. M6, M9).

[9] E. Rathakrishnan. Theoretical Aerodynamics. Wiley, 2013. DOI: 10.1017/S0001924000010629 (cit. on pp. M6, M8, M9).

\section{Author addresses}

1. B. J. Florio, CSIRO Mineral Resources, Australia; University of Western Australia, Australia. 
mailto:brendan.florio@uwa.edu.au orcid:0000-0001-6015-8537

2. R. McKibbin, Massey University, New ZEALAnd. mailto:r.mckibbin@massey . ac.nz

3. C. Braham, University of Western Australia, Australia. mailto:calum. braham@research.uwa.edu.au

4. J. R. Ockendon, University of Oxford, EnGLAND. mailto:ock@maths.ox.ac.uk

5. C. P. Please, University of Oxford, EnGLAnd. mailto:colin.please@maths.ox.ac.uk orcid:0000-0001-8917-8574 